\title{
Polymer Production by Plasma Polymerization of Oxygenated Organic Compounds
}

\author{
Antonio P. Nascimento Filho, Maria L. P. Silva \\ Depto. de Engenharia Elétrica, EPUSP \\ Nicole R. Demarquette \\ Depto. de Engenharia de Materiais, EPUSP
}

\begin{abstract}
This work was aimed at producing new polymeric materials using oxygenated organic compounds as main reactants and plasma enhanced chemical vapor deposition (PECVD) as a production technique. The films obtained were analyzed by profilometry, in order to determine the deposition rate, by Infrared Spectroscopy (FTIR) to identify the species deposited and by contact angle measurements to determine the polarity of the surface. For the reactants used, namely ethanol, acetone and 2-propanol, the plasma deposition process was least efficient with ethanol followed by acetone and 2-propanol. With the latter, reproducible thin films were obtained with a high deposition rate (up to $400 \AA / \mathrm{min}$ ). These films had a high amount of OH and could be wetted by common organic solvents, such as 2-propanol or acetone. The contact angle formed by drops of 2-propanol or acetone aqueous solution on the film produced from 2-propanol varied significantly with the concentration, from $0.9 \%$ to $50 \%$ by vol., indicating that the film is sensitive to polar solutions.
\end{abstract}

Keywords: Plasma, Polymerization and organic compounds.

\section{Introduction}

One of the major contributions from microelectronics to the science of surface protection is the deposition of very thin films, normally obtained by plasma enhanced chemical vapor deposition (PECVD). The possibility of modifying surfaces leads to new materials that may show novel, interesting mechanical and chemical characteristics, such as protection to oxidation and abrasion. These new films are usually required in Micro Electro Mechanical Structures (MEMS) for production of micro devices. For instance, devices based on micro channels can adsorb hydrocarbons from the air, an ability that may be used for environmental analysis ${ }^{[1]}$. Up to now, these devices are able only to concentrate non-polar compounds in a gaseous matrix. However, if the microchannel surfaces are modified, devices could be used for adsorption of polar compounds even in the liquid phase.

The film required to change the microchannels sur- face must be polar and present $\mathrm{OH}$ and/or $\mathrm{CO}$ bonds similar to the ones used in chromatographic columns ${ }^{[2]}$. The most appropriate reagents in this context are oxygenated organic compounds. Indeed, ethanol, acetone and 2-propanol are the best choices since they are environmentally friendly, non-toxic and low-cost reactants. This work is aimed at obtaining hydrophilic organic thin films by PECVD, which may be used in sensors for environmental control ${ }^{[3]}$. Although the plasma deposition of oxygenated organic compounds is possible, it has never been tried before ${ }^{[4,5]}$.

\section{Experimental}

Thin films were produced using a PECVD reactor described elsewhere ${ }^{[3]}$. It is a home-made $40 \mathrm{kHz}$ capacitively coupled reactor. The electrodes were used at room temperature. The films were deposited on Silicon Wafers P-type, $<100>, 10-20 \Omega \mathrm{cm}$, three inches in diameter. The films deposited were analyzed by profilometry, contact angle measurements, Infrared

Autor para correspondência: Antonio P. Nascimento Filho, Departamento de Engenharia Elétrica, EPUSP, Cidade Universitária, CEP: 05508-900, São Paulo, SP. E-mail: apnf@lsi.usp.br 


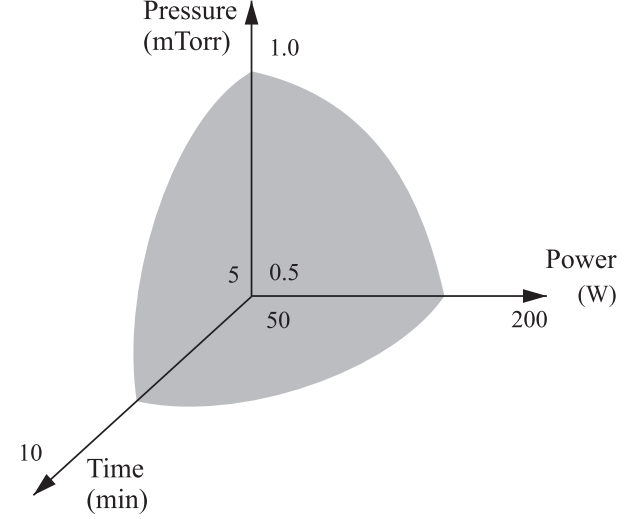

Figure 1. Process window used.

(FTIR) and Raman spectroscopy. Compatibility and chemical resistance to various organic compounds, inorganic acids and bases were tested. Ethanol, acetone and 2-propanol were used as reactants, which were expected to supply the oxygen species to ensure high polarity. The monomers were admitted in the chamber by differential pressure. Plasma polymerization was obtained with pressures in the reactor ranging from 0.5 to 2.0 Torr to ensure good plasma conditions, i.e. to avoid contamination from residual gases at lowpressure and particle formation at high pressures. The power ranged from $50 \mathrm{~W}$ to $200 \mathrm{~W}$, which correspond to the onset for plasma and the maximum value before electrode heating occurs, respectively. The time process was between 5 and 10 minutes. Figure 1 summarizes the process window.

In order to ensure a high amount of oxygen for the plasma reaction, the plasma reactor was modified inserting an auxiliary inlet gas system, located $10 \mathrm{~mm}$ from the substrate. The inlet gas system consisted of

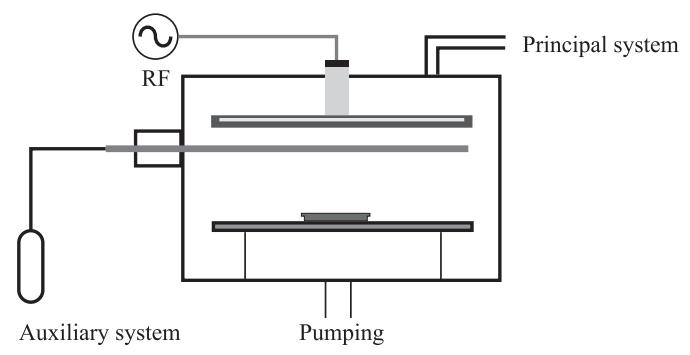

Figure 2a. Plasma chamber and the auxiliary system.

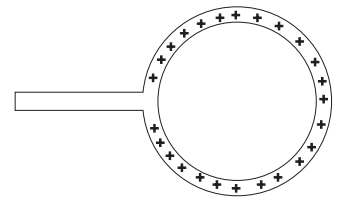

Figure 2b. Geometry of the stainless steel tube used as auxiliary system. a circular device, with a diameter of $200 \mathrm{~mm}$ formed by a $1 / 4 "$ circular stainless steel tube. Orifices were equally spaced by $10 \mathrm{~mm}$ from each other at the lower surface of the stainless steel tube. This system can be either powered (biased) or grounded. Figure $2 \mathrm{a}$ and $2 \mathrm{~b}$ show the plasma chamber and the auxiliary system.

Using this new inlet gas system it is possible to provide neutral species near the sample. Depositions were performed with grounded or powered auxiliary gas system. Positive bias was responsible for the most important changes in the plasma ${ }^{[1]}$. The maximum voltage used was $80.0 \mathrm{~V}$ due to the high instability of the plasma conditions when higher voltages are used.

\section{Results and Discussion}

An optimization procedure was initially adopted in order to select the best reactant to produce hydrophilic films, for which ethanol, acetone or 2-propanol were used as reactants.

\section{Ethanol and acetone depositions}

Although ethanol presented very stable plasmas, without plasmoid formation, it was not possible to obtain films under any of the conditions studied. Since powder formation was not observed, which means no reaction in vapor phase, the best explanation for the difficulties in obtaining films is that the active species formed are not able to polymerize. In order to learn whether the lack of polymerization was due to the low amount of species, the auxiliary inlet gas system was used and ethanol was admitted in the plasma reactor using both the conventional admittance and the auxiliary inlet-gas system. If the lack of polymerization occurred because of the physical and chemical characteristics of the species, the use of the inlet gas system would not affect the film-forming ability. The experimental results with the inlet gas system showed that it was possible to deposit a film with ethanol under the same pressures and power conditions, but with low deposition rates $(<100 \AA / \mathrm{min})$. Therefore, the lack of polymerization should be attributed to the small amount of species, which could not be sufficiently increased even when the auxiliary inlet gas system was used. Films were not formed because the amount of reactive species was too low, not because the species did not polymerize. This is consistent with results for diamond-like films ${ }^{[6]}$, in which high powers are necessary when ethanol is used and the amount of species formed is not limited by the deposition 


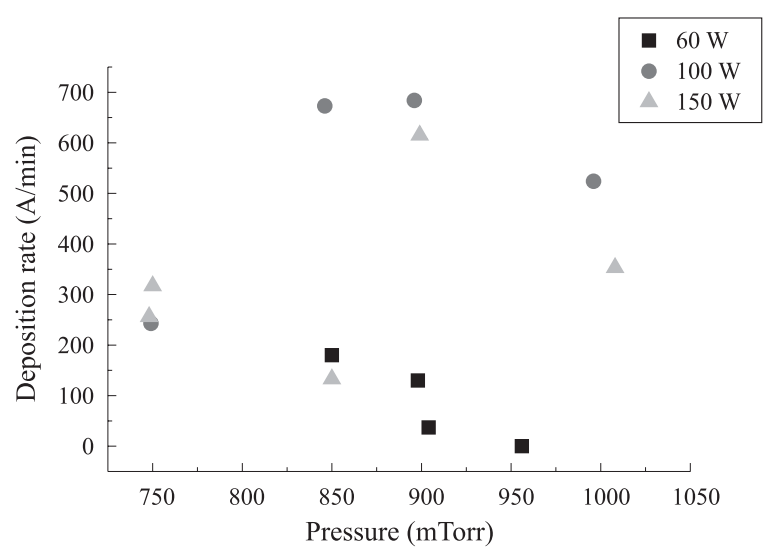

Figure 3. Deposition rate x pressure for different powers used for acetone reagent.

rate. In summary, ethanol is not a promising reagent to obtain plasma polymerized hydrophilic films.

Acetone proved to be a good reactant but powder formation was a critical issue. In fact, under any value of power applied, powder formation was observed at a pressure of approximately 1.0 Torr. The best conditions encountered were power $100 \mathrm{~W}$ and pressure 850 mTorr, as shown in Figure 3.

\section{2-propanol depositions}

Plasmas of 2-propanol were easily obtained, showing a high polymerization rate (ranging from 100 to $400 \AA / \mathrm{min}$ ) and a reproducible process. A maximum deposition rate was found for $150 \mathrm{~W}$ and 1.0 Torr, probably due to bombardment and powder formation. Figure 4 shows the maximum deposition rate obtained.

\section{Characterization}

FTIR analysis of ethanol, acetone and 2-propanol films showed $\mathrm{CH}_{\mathrm{n}}$ absorption, besides $\mathrm{OH}$ and $\mathrm{CO}$ under some conditions. The main difference is the relative intensity of each species present in the films. Typical FTIR spectra of the films deposited are shown

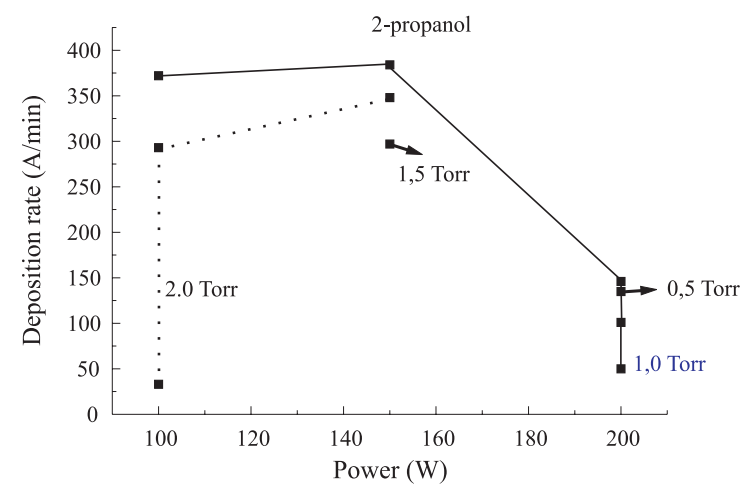

Figure 4. Deposition rate vs. power for different pressures studied. in figures 5, 6 and 7 when 2-propanol, acetone and ethanol were used, respectively.

The intensities of $\mathrm{OH}$ and $\mathrm{CO}$ are strongly dependent on the plasma conditions. High pressures and low powers favor their appearance. In order to understand the differences among the films, Raman Spectroscopy was performed, as shown in Figure 8. All films presented $\mathrm{CH}_{\mathrm{n}}$ absorptions, while for a few 2-propanol samples $\mathrm{C}=\mathrm{C}$ bonds also appeared, probably due to an easier dehydration of the monomer or active species for 2-propanol plasmas.

Some depositions of 2-propanol admitted in the plasma reactor using both the conventional admittance system and auxiliary inlet gas system were carried out in order to verify if the use of auxiliary inlet system, powered or grounded, could increase the amount of oxygenated species. When power was applied to the auxiliary system, the relative intensity of $\mathrm{CO}$ absorption increased. The results seem to indicate that the conventional system is responsible for the amount of $\mathrm{OH}$ species whereas the auxiliary system influences mainly the $\mathrm{CO}$ formation. The powered electrode favors oxidation of the carbon atom, probably due to secondary electron emission ${ }^{[7]}$.

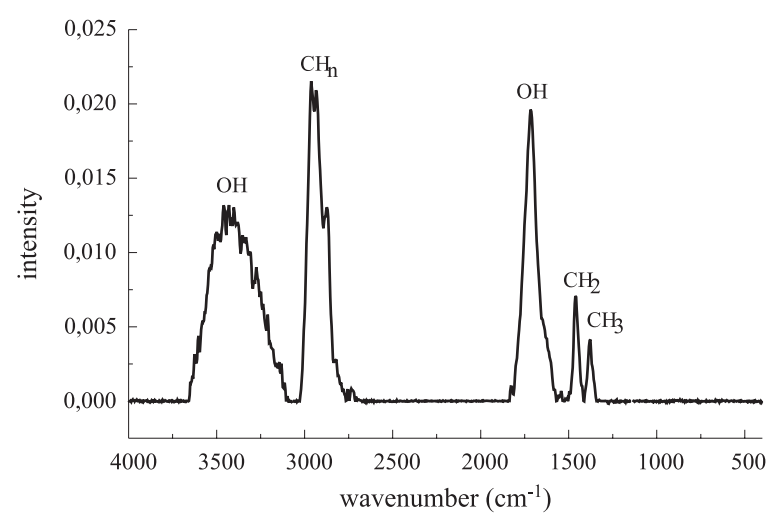

Figure 5. Typical FTIR spectrum obtained from 2-propanol reagent.

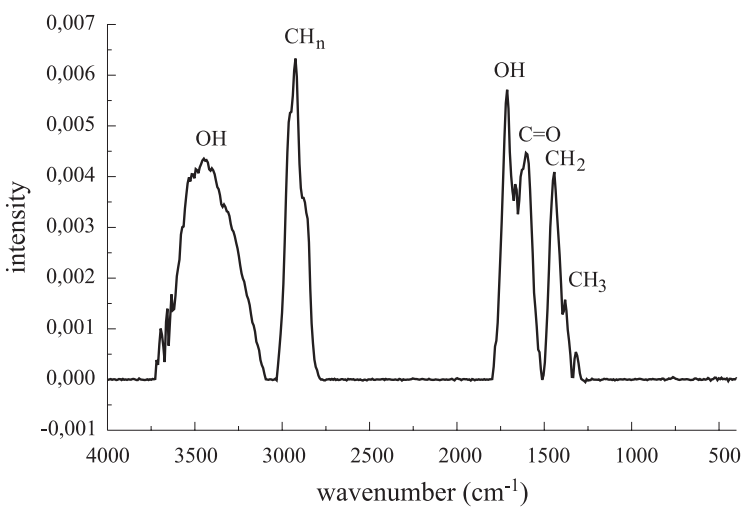

Figure 6. Typical FTIR spectrum obtained from acetone reagent. 


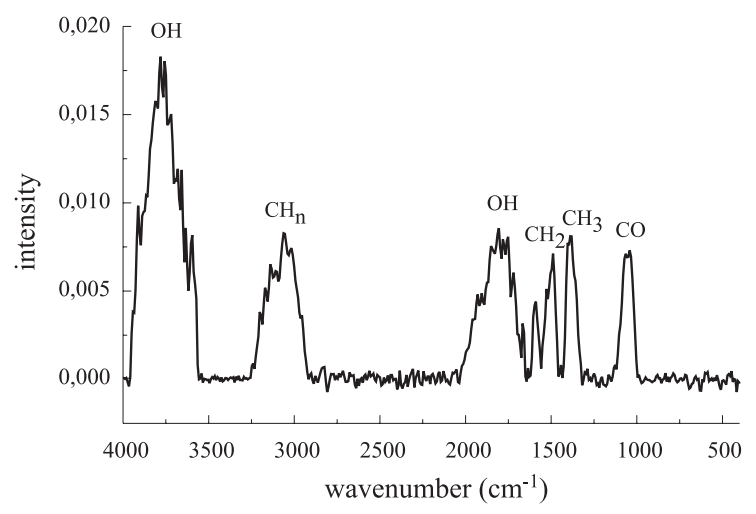

Figure 7. Typical FTIR spectrum obtained from ethanol reagent.

The best compromise to increase $\mathrm{OH}$ and $\mathrm{CO}$ simultaneously is the admittance of $10 \%$ of the total pressure of the 2-propanol by the auxiliary system.

The polymerization rate with 2-propanol monomer was strongly affected by using the auxiliary inlet gas system, mainly when voltage was applied $(80.0 \mathrm{~V})$. It is possible to obtain deposition rates from $375 \AA / \mathrm{min}$ to $550 \AA /$ min when injection flows between $10-90 \%$ of the total pressure through the auxiliary system is used.

FTIR analysis pointed out $\mathrm{CH}_{\mathrm{n}}, \mathrm{CH}_{2}, \mathrm{CH}_{3}, \mathrm{OH}$ and $\mathrm{C}=\mathrm{O}$ absorption. The increase in $\mathrm{C}=\mathrm{O}$ intensity occurs simultaneously with $\mathrm{OH}$ decrease, which seems to indicate that $\mathrm{C}=\mathrm{O}$ was formed at the expense of $\mathrm{OH}$ species.

Most films showed good resistance to acid and basic solutions. However, they could be peeled off when dipped in acetone (Figure 13). Aging of 2-propanol films showed that thicker films (more than 2400 A thick) can peel off after six months. Most likely the peeling caused by acetone can be attributed to a weak adhesion. The peeling that occurred in the aging test is most likely due to stresses present in the film, as shown in Figure 9.

In order to avoid peeling caused by acetone a double layer of HMDS/2-propanol film was deposited. It is well known that HMDS can improve adhesion ${ }^{[8]}$

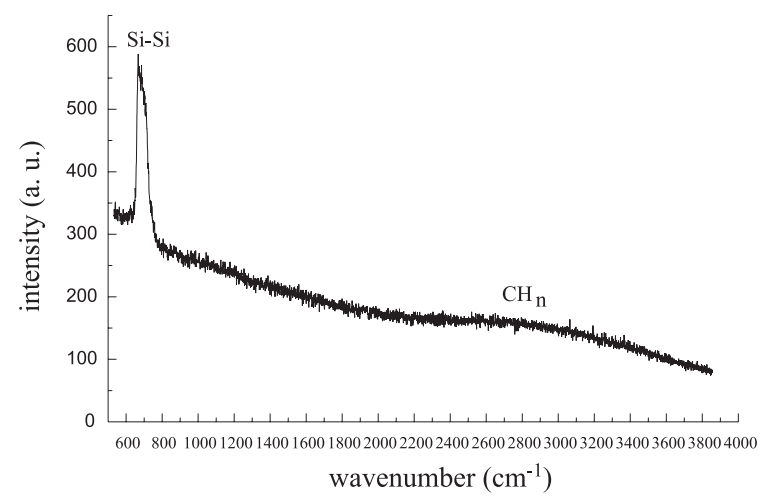

Figure 8. Typical Raman spectrum obtained from 2-propanol film.

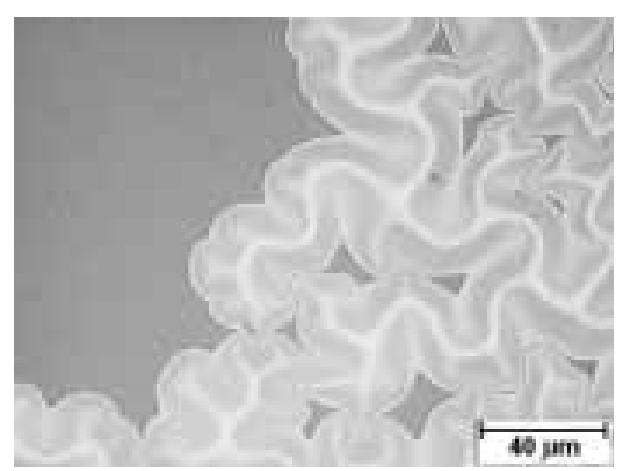

Figure 9. Photo obtained by optical microscopy showing stress

and that double layers can be a good solution to complex adhesion problem ${ }^{[8]}$. The new film formed was dipped in a $1 \mathrm{HF}: 1$ ethanol solution and proved to be resistant to peeling. These solutions were used because ethanol is a good solvent for polar organic compounds and HF is corrosive for inorganic compounds. Figures 10 and 11 show the film aspect before and after the use of HF solution, respectively.

The films obtained from 2-propanol and acetone monomers were hydrophilic, and could be wetted by water and organic liquids (2-propanol and acetone). However, acetone films showed contact angles higher than 2-propanol films. The contact angle measurements made by drops of water ranged from 66 to $77^{\circ}$ for acetone films and from 57 to $74^{\circ}$ for 2-propanol films, using or not the auxiliary inlet gas system. The contact angle measurements decrease exponentially with $\mathrm{OH} / \mathrm{CH}_{\mathrm{n}}$ FTIR relative intensity.

As the films showed good affinity to polar compounds, such as water and acetone, two samples were analyzed using contact angle measurements and different concentrations $(0.01-50 \%$ vol. $)$ of 2 propanol in acetone aqueous solution. Sample 1 was obtained using the conventional system and sample 2 using both the auxiliary inlet-gas and conventional admittance. Sample 1 shows a high $\mathrm{OH} / \mathrm{CHn}$ relative intensity and Sample 2 a high $\mathrm{CO} / \mathrm{CHn}$ relative

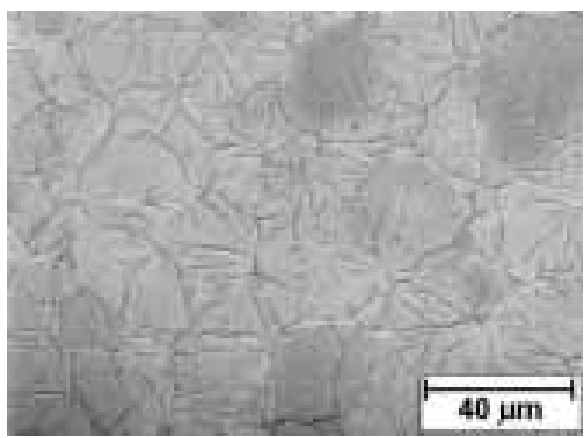

Figure 10. Film aspect before HF + ethanol solution 


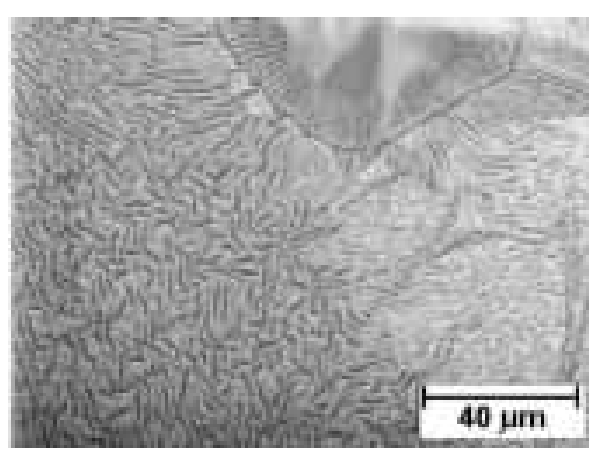

Figure 11. Film aspect after HF + ethanol solution

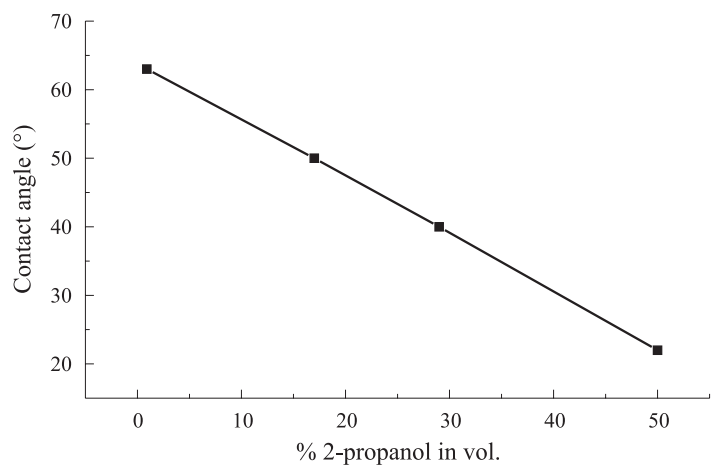

Figure 12. Contact angle measurement for 2-propanol sample using 2-propanol solution in different concentration $(0,9 \%$ to $50 \%$ in vol.).

intensity. Acetone solutions did not show large dispersion in contact angle measurements, ranging from $65^{\circ}$ to $57^{\circ}$ and from $67^{\circ}$ to $60^{\circ}$ for Sample 1and Sample 2, respectively. However, 2-propanol solutions showed a high and linear variation, ranging between $22^{\circ}$ and $65^{\circ}$, as shown in Figure 12.

Figures 13 and 14 show the film aspect, for sample one and two, after the use of acetone solution $0,01 \%$ vol. and after characterization with water, respectively. It can be noticed that the appearance of the film is changed even if only water is used. This seems to indicate that these films are similar to the ones used in chromatographic columns.

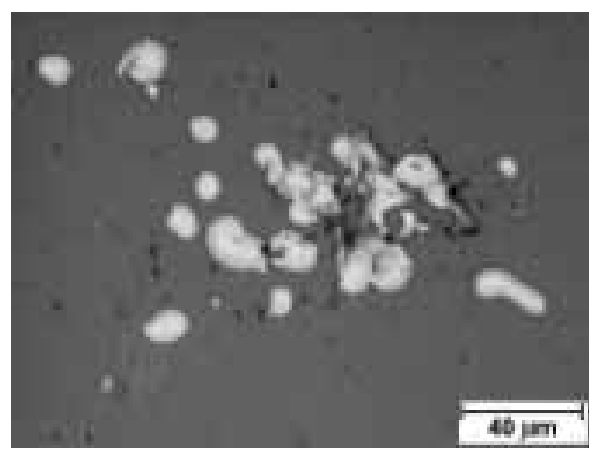

Figure 13. Photo obtained by optical microscopy for Sample 1 after the use of acetone solution $0.01 \%$ vol.

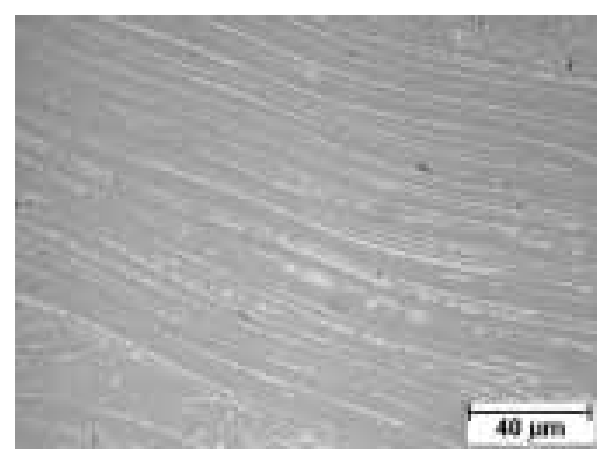

Figure 14. Photo obtained by optical microscopy for Sample 2 after the use of water solution.

\section{Conclusions}

Ethanol and acetone showed to be difficult for plasma polymerization and 2-propanol proved to be a good reactant to obtain high polar organic films. These films presented promising characteristics as adsorption layer for environmental sensors.

\section{Acknowledgements}

The authors would like to thank FAPESP for the financial support.

\section{References}

1. Micromechanical structures development for chemical analysis: study of the porous silicon as adsorbent, Souza, S. G. ; Galeazzo, E.; Silva, M. L. P. ; R. Furlan, R. and Fernandez, F. J. R., SPIE, number 3891-43, outubro, (1999).

2. Silva, M. L. P. ; Tan, I. H. - to be published in ISPC 2001.

3. Nogueira, S.; Silva, M. L. P. and Tan, I. H. - ISPC-14 Symposium proceedings, 1393 (1999).

4. Pan, Y. V.; Denton, D. D. - J. Appl. Polymer Sci, 7B, 17 (1999).

5. McTarggart, F. K.; and Elselvier, N. Y. - Pub. Co, (1967).

6. Watanabe, I. and Yoshie, K. - Jpn. J. Appl. Phys., 36, 792 (1997).

7. Silva, M. L. P. and Tan, I. H. - ISPC-15 Symposium proceedings, 1769 (2001).

8. Nogueira, S. - dissertação de Mestrado, Escola Politécnica da Universidade de São Paulo, (2000).

9. Chemical analysis columns and supplies, HP Catalog (1996)

10. Nogueira, S.; Silva, M. L. P. and Tan, I. H. - ISPC-14 Symposium proceedings, 1393 (1999).

Recebido: 10/08/01

Aprovado: 19/07/02 